solid solution at these concentrations of $\mathrm{Mn}$, was annealed for several hours at $500^{\circ} \mathrm{C}$. The echo amplitude increased by about a factor 2 while $T_{2}$ remained unchanged. $\mathrm{Cw}$ absorption linewidths ${ }^{4}$ were observed to sharpen by about $20 \%$ after similar anneals. It may be that the impurities have sufficient energy at the annealing temperature to diffuse to grain boundaries or to cluster, effectively reducing the impurity concentration in the silver matrix. The noted increase in echo amplitude is then consistent with the observation that smaller impurity concentrations yield larger echoes. This does not rule out explanations based on the removal of dislocations.

The measurements were made using a fixedfrequency Bruker pulse spectrometer, phase-coherent pulses, a single-coil probe, and diode detection.

Echoes have been reported in (a) nuclear paramagnetic resonance, ${ }^{1}$ (b) ESR, ${ }^{6}$ (c) optical transitions in atoms ${ }^{7}$ (d) ferrimagnetic resonance, ${ }^{8}$ (e) free electrons in a plasma, ${ }^{9}$ and (f) type-II superconductors. ${ }^{2}$

While the Ag NMR is expected to be severely inhomogeneously broadened (approximately $20 \mathrm{kG}$ for first neighbors) due to the presence of local moments on the Mn ions, the signals observed here are (ruling out an unlikely enhancement mechanism) far too intense to be due to the usual nuclear spin echoes. ${ }^{1}$ (Spin echoes in pure $\mathrm{Ag}$ and $\mathrm{Ag}-\mathrm{Pd}$ compounds ${ }^{10}$ are only observable, for example, using signal-processing techniques, superconducting field strengths, and liquid-helium temperatures.) In addition, the decay constant is 150 times shorter that the spin dephasing time in pure silver metal. ${ }^{11}$ The requirement that the radiating source have a strong component at the
4- $\mathrm{MHz}$ detection frequency militates against explanation of the phenomenon by (b)-(e) above. Furthermore, the time scale in this process is tens of microseconds whereas in (b)-(e) it is nanoseconds.

There is a striking similarity between the major characteristics of the echoes reported here and those recently reported in superconductors. ${ }^{2}$ Properties (2)-(4) and perhaps (5) and (6) are, qualitatively if not quantitatively, essentially the same. If these echoes have a common source, then they are not associated with the superconducting state and it is possible that they are a more widespread phenomenon than heretofore realized. Experiments are in progress to see if similar echoes exist in other alloy systems in an attempt to gain more information about their origin.

We are grateful to Handy and Harman for kindly providing some of the alloy samples and analyses.

\footnotetext{
${ }^{1}$ E. L. Hahn, Phys。Rev. $\underline{80}, 580$ (1950).

${ }^{2}$ I. B. Goldberg, E. Ehrenfreund, and M. Weger, Phys. Rev. Letters 20, 539 (1968).

${ }^{3}$ G. F. Herrmann and R. F. Whitmer, Phys. Rev. 143, 122 (1966).

${ }^{4}$ R. J. Snodgrass, to be published.

${ }^{5}$ W. B. Mims, Phys. Rev. 141, 499 (1966).

${ }^{6} \mathrm{~J}$. P. Gordon and K. D. Bowers, Phys. Rev. Letters 1, 368 (1958).

${ }^{7}$ N. A. Kurnit, I. D. Abella, and S. R. Hartmann, Phys. Rev. Letters $\underline{13}$, 567 (1964).

${ }^{8}$ D. E. Kaplan, Phys. Rev. Letters 14, 254 (1965)。

${ }^{9}$ R. M. Hill and D. E. Kaplan, Phys. Rev. Letters 14 , 1062 (1965).

${ }^{10}$ A. Narath, J. Appl. Phys. 39, 553 (1968).

${ }^{11}$ A. Narath, A. T. Fromhold, Jr., and E. D. Jones, Phys. Rev. 144, 428 (1966).
}

\title{
SELF-FOCUSING OF LIGHT BY POTASSIUM VAPOR
}

\author{
D. Grischkowsky \\ IBM Thomas J. Watson Research Center, Yorktown Heights, New York 10598 \\ (Received 24 February 1970)
}

Raman-shifted ruby-laser light on the high-frequency side of the ${ }^{2} P_{3 / 2}$ resonance line $(7665 \AA)$ of potassium was self-focused by a dilute potassium gas.

Previous studies of self-focusing and self-trapping of light have been complicated by lack of knowledge of the constitutive relationship between the applied field and the induced polarization. $^{1-5}$ Also, other nonlinear effects occur when the fields reach the high values characteristic of self-focused beams and these broaden the frequency and deplete the power of the beams. ${ }^{4,5}$
However, on the wings of a resonance line of a dilute gas, the constitutive relation is known, and the other nonlinear effects should be absent. This paper reports the observation of self-focusing of light due to the intensity-dependent dispersion of a resonance line, a possibility which has been considered theoretically by Javan and Kelley. ${ }^{6}$ As shown in Figs。 1 and 2, Raman-shifted 


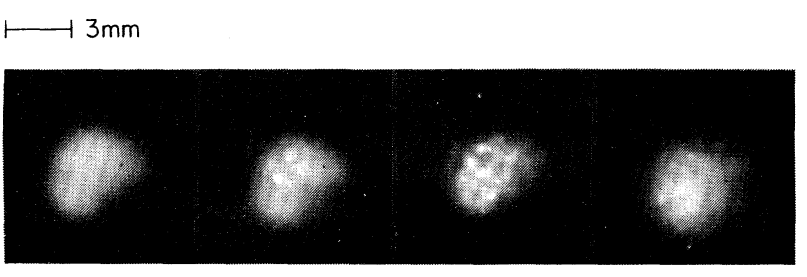

$160^{\circ} \mathrm{C} \quad 170^{\circ} \mathrm{C} \quad 190^{\circ} \mathrm{C} \quad 210^{\circ} \mathrm{C}$

FIG. 1. Photographs of the Raman beam at the exit window of the $100-\mathrm{cm}$ potassium cell versus cell temperature. The frequency of the inhomogeneous $1-\mathrm{mm}-$ diam beam was $12 \mathrm{~cm}^{-1}$ above the frequency of the $7665-\AA$ potassium line, and the beam had a peak power of $10 \mathrm{~kW}$ and a pulse width of $5 \mathrm{nsec}$.

ruby-laser light on the high-frequency side of the ${ }^{2} P_{3 / 2}$ resonance line (7665 $\AA$ ) was self-focused by a dilute potassium gas. The conditions under which self-focusing occurred agree with theory.

The ruby laser was $Q$ switched by vanadium phthalocyanine in nitrobenzene and produced a 20MW, 15-nsec pulse with a linewidth of approximately $0.02 \mathrm{~cm}^{-1}$. The laser wavelength could be tuned over a $6-\mathrm{cm}^{-1}$ range by changing the ruby temperature. The Raman liquid was either $m$-fluoronitrobenzene (Raman shift $1353 \mathrm{~cm}^{-1}$ ) or nitrobenzene (Raman shift $1345 \mathrm{~cm}^{-1}$ ). The Raman-shifted output pulse was separated from the ruby-laser light by two 7-69 Corning filters. Megawatt Raman pulses with linewidths of the order of $1 \mathrm{~cm}^{-1}$ could be easily obtained from either liquid. The Raman beam traversed a 100$\mathrm{cm}$ potassium cell and photographs of the beam at the exit window were taken as a function of cell temperature. The given temperature describes approximately the central $50 \mathrm{~cm}$ of the cell. The cell windows were kept $\sim 20^{\circ} \mathrm{C}$ hotter than the cell center to prevent fogging. Polaroid type 413 infrared film was used, which is a highcontrast emulsion with a small dynamic range. Using calibrated neutral density filters, we could easily make relative measurements of the energy per unit area of the output beam. Raising the temperature changes the dielectric constant $\epsilon$, because the atomic number density $N$ increases.

Figure 1 shows self-focusing of a multitransverse-mode Raman beam from nitrobenzene. The frequency of the beam was approximately 12 $\mathrm{cm}^{-1}$ higher than the potassium resonance and hence was far out on the Lorentzian wings of the line. For the cold cell the beam had a $1-\mathrm{mm}$ diameter at the input window and a $3-\mathrm{mm}$ diameter at the exit window of the $100-\mathrm{cm}$ potassium cell. The peak power of the 5-nsec Raman pulse was $\longmapsto 1 \mathrm{~mm}$

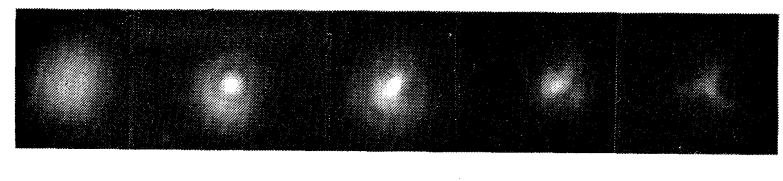

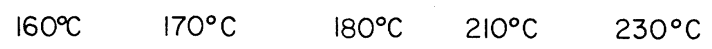

FIG. 2. Photographs of the Raman beam at the exit window of the $100-\mathrm{cm}$ potassium cell versus cell temperature. The frequency of the nearly diffraction-limited $1-\mathrm{mm}$-diam beam was $12 \mathrm{~cm}^{-1}$ above the frequency of the $7665-\AA$ potassium line, and the beam had a peak power of $1.5 \mathrm{~kW}$ and a pulse width of $10 \mathrm{nsec}$.

$10 \mathrm{~kW}$. For temperatures lower than $160^{\circ} \mathrm{C}$ no self-focusing was observed with this beam. At $165^{\circ} \mathrm{C}$ bright spots began to appear in the exit beam. The number and the relative intensity of the spots increased with temperature, with some spots becoming surrounded by rings. The observed pattern was different for each laser flash, but the general behavior was reproducible.

Above $190^{\circ} \mathrm{C}$ the spots began to disappear with rising temperature, and at $210^{\circ} \mathrm{C}$ the effect was greatly reduced. It appears as if different sections of the inhomogeneous input beam were independently self-focused and then defocused with increasing temperature. If self-trapping had occurred, the number and relative intensity of the spots of light would have been expected to increase monotonically with temperature.

Figure 2 shows self-focusing of a nearly diffraction-limited Raman beam from nitrobenzene. The frequency of the beam was approximately 12 $\mathrm{cm}^{-1}$ higher than the potassium resonance, and the peak power during the irregular 10-nsec pulse was $1.5 \mathrm{~kW}$. The 1 -mm-diam beam was obtained by focusing the Raman light on a 250$\mu \mathrm{m}$-diam pinhole at the focus of a $20-\mathrm{cm}$ focal length lens having an aperture of $1 \mathrm{~mm}$. Again, for temperatures lower than $160^{\circ} \mathrm{C}$, no self-focusing was observed. At $170^{\circ} \mathrm{C}$ self-focusing occurred; the exit beam was composed of a central core surrounded by a diffuse ring. The diameter $d_{c}$ of the core was approximately $100 \mu \mathrm{m}$, and the energy per unit area $U_{c}$ of the core was about 3 times the energy per unit area $U_{0}$ of the unfocused beam. This first appearance of the bright core indicated that at peak power the "self-focusing length" ${ }^{3} Z_{f}$ was equal to the length of the potassium cell. For temperatures between 170 and $200^{\circ} \mathrm{C}$ the exit beam always contained a central core, which was surrounded by a diffuse ring-type structure. Sometimes more than one 
core was present, which appeared to be due to some inhomogeneous transverse structure of the input beam. At a given temperature the general features of the core were reproducible, but the detailed beam pattern varied. At $180^{\circ} \mathrm{C}, d_{c} \approx 75$ $\mu \mathrm{m}$ and $U_{c} \approx 30 U_{0} ;$ at $190^{\circ} \mathrm{C}, d_{c} \approx 50 \mu \mathrm{m}$ and $U_{c}$ $\approx 30 U_{0}$; at $200^{\circ} \mathrm{C}, d_{c} \approx 75 \mu \mathrm{m}$ and $U_{c} \approx 6 U_{0}$. At $210^{\circ} \mathrm{C}$ the center of the beam was still the most intense, but the central core had vanished. At $230^{\circ} \mathrm{C}$ the entire beam started to disappear. The focusing action of the vapor was quite strong at 180 and $190^{\circ} \mathrm{C}$; the ratios of $U_{c}$ to $U_{0}$ of $30: 1$ give only a lower limit to the intensity of the core, as the core is not expected to exist for the entire pulse. It is not clear what mechanism caused loss of the core as the temperature was raised above $190^{\circ} \mathrm{C}$. If the core corresponded to selftrapping, one would expect $U_{c}$ to increase continually and $d_{c}$ to decrease continually as the temperature was raised above $170^{\circ} \mathrm{C}$. The exit window would intersect the self-trapped filament for the time the power of the input beam exceeded that required for a self-focusing distance of $100 \mathrm{~cm}$. Our results are more consistent with those expected from a focus moving in response to the changing power of the pulse. ${ }^{7-9}$ Because of the appearance of the core over a $30^{\circ} \mathrm{C}$ interval, the focal region appears to extend for many centimeters in the direction of beam propagation. $Z_{f}$ changes $^{6}$ as $N^{-1 / 2}$; since $Z_{f}=100 \mathrm{~cm}$ at $170^{\circ} \mathrm{C}$ where $N=4 \times 10^{13}$ atoms $/ \mathrm{cm}^{3},{ }^{10}$ at $190^{\circ} \mathrm{C}$ where $N$ $=10^{14}$, we calculate $Z_{f}=65 \mathrm{~cm}$. The power loss of the beam was presumably due to the subsequent defocusing, which occurred beyond the region of the cell where the beam was self-focused. Although the steady-state absorption coefficient $\alpha$ is not applicable to our pulsed experiment, it does give an upper limit for the absorption by the potassium vapor. For potassium vapor at $230^{\circ} \mathrm{C}$ and for a frequency $12 \mathrm{~cm}^{-1}$ away from the $7665-\AA$ resonance line, $\alpha \approx 2.5 \times 10^{-3} \mathrm{~cm}^{-1}$; this value includes the dominating effect of collision broadening. ${ }^{11}$

Using $m$-fluoronitrobenzene as the Raman liquid and changing the temperature of the ruby, we were able to make observations with the frequency $\nu$ of the Raman beam $4 \mathrm{~cm}^{-1}$ above, 2 $\mathrm{cm}^{-1}$ above, and $2 \mathrm{~cm}^{-1}$ below the frequency $\nu_{0}$ of the 7665- $\AA$ line of potassium. Self-focusing was observed when $\nu_{0}<\nu$ and was not observed when $\nu<\nu_{0}$. When $\nu<\nu_{0}$, some of our results suggest that the beam was self-defocused, and experiments are in progress to clarify this point. For the self-focusing observations made with $m$-fluoronitrobenzene a $1-\mathrm{mm}$-diam diffractionlimited beam with a peak power of $400 \mathrm{~W}$ and a pulse width of 5 nsec was used. For self-focusing, $U_{c} / U_{0}$ increased as $\nu-\nu_{0}$ increased, and the temperature interval over which the core existed increased as $\nu-\nu_{0}$ increased. With $\nu-\nu_{0}$ $=2 \mathrm{~cm}^{-1}$, the self-focusing action of the vapor was already apparent at $90^{\circ} \mathrm{C}$, and $Z_{f} \approx 100 \mathrm{~cm}$ at $130^{\circ} \mathrm{C}$. For $\nu-\nu_{0}=4 \mathrm{~cm}^{-1}, Z_{f} \approx 100 \mathrm{~cm}$ at $150^{\circ} \mathrm{C}$. In both cases the beam suffered a noticeable energy loss for temperatures below that required for $Z_{f} \approx 100 \mathrm{~cm}$. When the temperature was raised above the point at which $Z_{f}=100 \mathrm{~cm}$, the beam expanded, and the beam energy was further reduced.

The dielectric response of the potassium vapor to an intense pulse of near-resonant light is obtained from consideration of the precession of the pseudo-electric dipole moment about the pseudo-electric field of the pulse. ${ }^{12}$ For the experiments shown in the figures the frequency of the pulse is approximately $12 \mathrm{~cm}^{-1}$ away from the resonant frequency. Consequently, the precession frequency $\Delta$ of the pseudomoment about the effective field in the rotating frame, the coordinate frame in which the pseudofield appears stationary, is of the order of $3.6 \times 10^{11} \mathrm{~Hz}$. Since the electric field of the pulse changes slowly compared with $\Delta$, the effective field changes adiabatically, ${ }^{13}$ and the pseudomoment will remain aligned along the changing effective field. Furthermore, the magnitude of the pseudomoment will not change for times short compared with $T_{1}$. In the absence of radiative trapping, $T_{1}$ is the radiative lifetime of the excited state $(26$ nsec for the 7665- $\AA$ line of potassium). This behavior is related to the situation in NMR, where the magnetization becomes aligned along the effective field direction in the rotating frame for high, near-resonant, rf fields. ${ }^{13,14}$ For pulses short compared with $T_{1}$ and for amplitude changes which occur slowly compared with $\Delta$, the dielectric constant $\epsilon$ for the potassium vapor is obtained from the geometrical model given above as

$$
\epsilon=1-4 \pi N p_{12}^{2} /\left[h\left(\nu-\nu_{0}\right)\left(1+E^{2} / E_{s}{ }^{2}\right)^{1 / 2}\right] .
$$

$N$ is the number density of atoms; $E$ is the field strength of the incident linearly polarized light; $p_{12}=6.2 \times 10^{-18}$ esu is the matrix element of the dipole moment along the field direction; $\nu$ is the frequency of the incident light; $\nu_{0}$ is the frequency corresponding to the energy separation between the ${ }^{2} P_{3 / 2}$ and ${ }^{2} S_{1 / 2}$ levels; and $E_{s}=\left|h\left(\nu-\nu_{0}\right)\right|$ $p_{12} l_{0}$ 
The equation for $\epsilon$ is not the standard steadystate result of the Bloch equations as given by Javan and Kelley. $T_{1}$ and $T_{2}$ do not appear in our result for $\epsilon$, and $\epsilon$ saturates as $E^{-1}$ rather than as $E^{-2}$ for the steady-state result. However, for $T_{2}=2 T_{1}$ the steady-state solution yields

$$
\epsilon=1-4 \pi N p_{12}^{2} /\left[h\left(\nu-\nu_{0}\right)\left(1+E^{2} / 2 E_{s}^{2}\right)\right],
$$

which agrees as well with experiment as does our result. Short-pulse experiments are in progress which should be capable of distinguishing between the two expressions for $\epsilon$ with their different saturation behavior.

The index of refraction $n$ of the vapor is $\epsilon^{1 / 2}$. When $E \ll E_{s}, n$ can be written in the form $n=n_{0}$ $+\delta n$, where $n_{0}$ is the normal index of the vapor and $\delta n$ is the intensity-dependent change in the index,

$$
\delta n=\left[\pi N p_{12}{ }^{4} / h^{3}\left(\nu-\nu_{0}\right)^{3}\right] E^{2} .
$$

Javan and Kelley give the expression $Z_{f}=d\left(n_{0}\right)$ $16 \delta n)^{1 / 2}$ for the self-focusing length, where $d$ is the diameter of the input beam and $\delta n$ is the index change caused by the input beam. Considering the data of Fig. 2, we set $Z_{f}=100 \mathrm{~cm}$, and using $E^{2}$ corresponding to $1.5 \mathrm{~kW}$, we calculate $N=10^{14}$ and obtain the corresponding temperature of $190^{\circ} \mathrm{C}$. This is in reasonably good agreement with the experimental value of $170^{\circ} \mathrm{C}$ considering the irregular pulse shape and the imprecise data available for $N$ vs $T$ of potassium vapor. ${ }^{10}$ If we assume complete saturation of $\epsilon$ in the core at $170^{\circ} \mathrm{C}$, the measured diameter of $100 \mu \mathrm{m}$ can be compared with a calculated value. Javan and Kelley give the relationship $d_{\text {min }}$ $=1.22 \lambda\left(8 n_{0} \delta n_{\text {max }}\right)^{-1 / 2}$ for the minimum beam diameter, if there is a maximum possible change $\delta n_{\max }$ in the index. The equation for $\epsilon$ yields $\delta n_{\text {max }}=2 \pi N p_{12}^{2} / h\left(\nu-\nu_{0}\right)$. Given $N=4 \times 10^{13}$ at $170^{\circ} \mathrm{C}, d_{c}$ is calculated to be $160 \mu \mathrm{m}$, which is significantly larger than the measured value.
However, $d_{\min }$ is the diameter of the lowest order self-trapped mode, and if self-trapping does not occur $d_{\min }$ will only be an estimate for $d_{C}$.

The many discussions with J. A. Armstrong concerning the form of $\epsilon$ used in this paper and the interpretation of the data are gratefully acknowledged. I would like to thank P. P. Sorokin for many invaluable comments concerning the outlines of the experiment and various aspects of the results. The expert technical assistance of T. G. Kazyaka in setting up the experiment and the taking of data is greatly appreciated.

\footnotetext{
${ }^{1}$ G. A. Askar'yan, Zh. Eksperim. i Teor. Fiz. $\underline{42}$, 1567 (1962) [Soviet Phys. JETP 15, 1088 (1962)].
}

${ }^{2}$ R. Y. Chiao, E. Garmire, and C. H. Townes, Phys. Rev. Letters 13, 479 (1964).

${ }^{3}$ P. L. Kelley, Phys. Rev. Letters 15, 1005 (1965).

${ }^{4}$ The recent literature is summarized in the review article by S. A. Akhmanov, A. P. Sukhorukov, and R. V. Khokhlov, Usp. Fiz. Nauk 93, 19 (1967) [Soviet Phys. Usp. 10, 609 (1968)].

${ }^{5}$ R. G. Brewer, J. R. Lifsitz, E. Garmire, R。Y. Chiao, and C. H. Townes, Phys. Rev. 166, 326 (1968).

${ }^{6}$ A. Javan and P. L. Kelley, IEEE J. Quantum Electron. 2 , 470 (1966).

${ }^{7}$ V. N. Lugovoi and A. M. Prokhorov, Zh. Eksperim. i Teor. Fiz.-Piz'ma Redakt. 7, 153 (1968) [JETP Letters 7,117 (1968)].

${ }^{8}$ E. L. Dawes and J. H. Marburger, Phys. Rev. 179, 862 (1969).

${ }^{9}$ M. M. T. Loy and Y. R. Shen, Phys. Rev. Letters $\underline{22}$, 994 (1969) .

${ }^{10}$ A. N. Nesmeyanov, Vapour Pressure of the Elements (Academic, New York 1963).

${ }^{11}$ D. S. Hughes and P. E. Lloyd, Phys. Rev. $\underline{52}, 1215$ (1937) .

${ }^{12}$ I. D. Abella, N. A. Kurnit, and S. R. Hartmann, Phys. Rev.141, 391 (1966).

${ }^{13} \mathrm{~A}$. Abragam, The Principles of Nuclear Magnetism (Oxford Univ., London, 1961).

${ }^{14}$ A. G. Redfield, Phys. Rev. 98, 1787 (1955). 

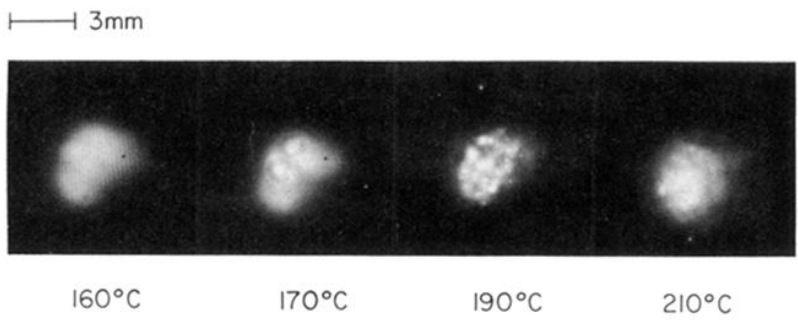

FIG. 1. Photographs of the Raman beam at the exit window of the $100-\mathrm{cm}$ potassium cell versus cell temperature. The frequency of the inhomogeneous $1-\mathrm{mm}-$ diam beam was $12 \mathrm{~cm}^{-1}$ above the frequency of the $7665-\AA$ potassium line, and the beam had a peak power of $10 \mathrm{~kW}$ and a pulse width of $5 \mathrm{nsec}$. 


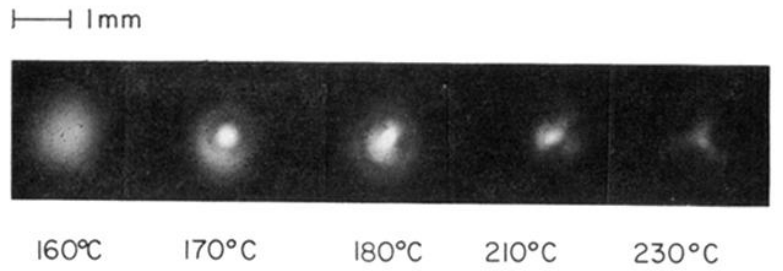

FIG. 2. Photographs of the Raman beam at the exit window of the $100-\mathrm{cm}$ potassium cell versus cell temperature. The frequency of the nearly diffraction-limited $1-\mathrm{mm}$-diam beam was $12 \mathrm{~cm}^{-1}$ above the frequency of the $7665-\AA$ potassium line, and the beam had a peak power of $1.5 \mathrm{~kW}$ and a pulse width of $10 \mathrm{nsec}$. 